\title{
A VARIANT OF SEPARABILITY IN DUAL SYSTEMS
}

\author{
by J. O. POPOOLA† and I. TWEDDLE
}

(Received 19th November 1975)

\section{Introduction}

In (12) we introduced the concept of essential separability and used it to define two classes of locally convex spaces, $\delta$-barrelled spaces and infra- $\delta$ spaces, which serve as domain and range spaces respectively in certain closed graph theorems (12, Theorems 3 and 7). In this note we continue the study of these ideas. The relevant definitions are reproduced below.

Section 2 is concerned with characterisations of essential separability and its connection with weak compactness properties. In Section 3 we discuss some relationships between $\delta$-barrelled, barrelled and countably barrelled spaces. Finally, in Section 4 we consider the associated $\delta$ barrelled topology of an infra- $\delta$-space in connection with a completeness result of $\mathrm{V}$. Eberhardt and $\mathrm{N}$. Adasch for infra-s-spaces.

Generally we follow the topological vector space notation of (13). Except where alternative symbols are introduced in the text, $E^{*}$ will denote the algebraic dual of a vector space $E$, and when $E$ is a separated locally convex space, $E^{\prime}$ will represent its (continuous) dual. When we refer to the dimension of $E(\operatorname{dim} E)$ we shall always mean its vector space dimension. $\left.\xi\right|_{A}$ denotes the induced topology on a subset $A$ of a topological space $(X, \xi),|B|$ is the cardinality of a set $B$ and $c$ is the cardinal number of the real field.

We are grateful to the referee for improving our original version of Theorem 2, which now appears as a corollary.

\section{Essentially separable sets}

We begin by reformulating the definition of essential separability which was given in (12). Let $(E, F)$ be a dual pair. We regard $E$ as a subspace of $F^{*}$ and say that a subset $A$ of $E$ is essentially separable for the dual pair $(E, F)$ if it is contained in a $\sigma\left(F^{*}, F\right)$-separable set. When the dual pair is clearly indicated, we simply say that $A$ is essentially separable. In particular, if $E$ is a separated locally convex space and $A$ and $B$ are subsets of $E$ and $E^{\prime}$ respectively, we will usually write " $A$ (resp. $B$ ) is essentially

$\dagger$ The first author was supported by a Commonwealth Academic Staff Scholarship which he gratefully acknowledges. 
separable" for " $A$ (resp. $B$ ) is essentially separable for the dual pair ( $\left.E, E^{\prime}\right)$ (resp. $\left.\left(E^{\prime}, E\right)\right)$ ".

Theorem 1. If $A$ is essentially separable for the dual pair $(E, F)$ then $\left.\sigma(E, F)\right|_{A}$ has a base consisting of at most $c$ sets.

Proof. This is trivial if $A=\emptyset$. Otherwise let $H$ be the linear span of $A$ and let $G$ be the $\sigma\left(F^{*}, F\right)$-closed linear span of $A$. Since $\left(F / H^{o}\right)^{*}$ is isomorphic to $G$ and $G$ is $\sigma\left(F^{*}, F\right)$-separable (12, Corollary to Theorem 1), it follows that $\left(F / H^{o}\right)^{*}$ is isomorphic to a product of at most $c$ copies of the scalar field (4, Chapter VIII, Theorem 7.2). Thus the dimension and consequently the cardinality of $F / H^{\circ}$ are at most $c$. Let $\Phi$ be the set of all non-empty finite subsets of $F / H^{\circ}$, so that $|\Phi| \leqslant c$, and let $\left\{x_{n}: n \in N\right\}$ be an at most countable $\sigma\left(F^{*}, F\right)$-dense subset of $G$. Note that $\sigma\left(F^{*}, F\right)$, $\sigma\left(G, F / H^{o}\right)$ and $\sigma(E, F)$ all coincide on $A$.

Let

$$
\mathscr{T}=\left\{\left\{x \in A:\left|\left\langle x-x_{n}, x^{\prime}\right\rangle\right|<1, x^{\prime} \in \phi\right\}: \phi \in \Phi, n \in \mathbb{N}\right\}
$$

Certainly $|\mathscr{T}| \leqslant c$ and each element of $\mathscr{T}$ is $\left.\sigma(E, F)\right|_{A^{-}}$open. Let $y \in A$ and let $U$ be any $\left.\sigma(E, F)\right|_{A}$-neighbourhood of $y$. There exists $\phi_{0} \in \Phi$ such that

$$
V=\left\{x \in A:\left|\left\langle x-y, x^{\prime}\right\rangle\right|<1, x^{\prime} \in \phi_{0}\right\} \subseteq U .
$$

Also there exists $n_{0} \in \mathbf{N}$ such that

$$
x_{n_{0}} \in\left\{x \in G:\left|\left\langle x-y, x^{\prime}\right\rangle\right|<1, x^{\prime} \in 2 \phi_{0}\right\} .
$$

Then $W=\left\{x \in A:\left|\left\langle x-x_{n_{0}}, x^{\prime}\right\rangle\right|<\mathbf{1}, \quad x^{\prime} \in 2 \phi_{0}\right\} \in \mathscr{T}, \quad y \in W$ and $W \subseteq V \subseteq$ $U$. Thus $\mathscr{T}$ is a base for $\left.\sigma(E, F)\right|_{A}$.

Since any topological space has a dense subset of cardinality at most that of a given base for its topology, we have immediately:

Corollary. If $A$ is essentially separable for the dual pair $(E, F)$, then $A$ has a $\sigma(E, F)$-dense subset of cardinality at most $c$.

The next result and its corollary are analogues of (9, Proposition 1.3).

Theorem 2. Let $E$ be a topological vector space with topology $\xi$, let $A$ be an absolutely convex subset of $E$ and let $\mathscr{A}$ be a base of neighbourhoods of 0 for $\left.\xi\right|_{A}$. For each $W \in \mathscr{A}$, let $W^{\prime}$ be an open balanced $\xi$-neighbourhood of 0 such that $W^{\prime} \cap A \subseteq W$. Then if $D$ is a dense subset of $A$, the sets $\left(d+W^{\prime}\right) \cap A(d \in D, W \in \mathscr{A})$ form a base for $\left.\xi\right|_{A}$.

Proof. Let $y \in A$ and let $Y$ be any $\left.\xi\right|_{A}$-neighbourhood of $y$. There exist $\xi$-neighbourhoods $U$ and $V$ of 0 and $W \in \mathscr{A}$ such that

$$
(y+U) \cap A \subseteq Y, \quad V+V+V \subseteq U \text { and } W \subseteq V(*)
$$

Choose $d \in\left(y+\left(V \cap W^{\prime}\right)\right) \cap D$ and let $x \in X=\left(d+W^{\prime}\right) \cap A$. Since $A$ is 
absolutely convex and $W^{\prime}$ is balanced, $\frac{1}{2}(x-d) \in A \cap W^{\prime}$ and so by $(*)$

$$
x=y+2\left(\frac{1}{2}(x-d)\right)+(d-y) \in(y+2 W+V) \cap A \subseteq Y .
$$

The result now follows since $X$ is an $\left.\xi\right|_{A}$-open set which contains $y$.

Corollary. Let $(E, F)$ be a dual pair and let $A$ be an absolutely convex subset of $E$. Then $\left.\sigma(E, F)\right|_{A}$ has a base consisting of at most $c$ sets if and only if

(i) 0 has a base of neighbourhoods for $\left.\sigma(E, F)\right|_{A}$ consisting of at most $c$ sets,

(ii) A has a $\sigma(E, F)$-dense subset of cardinality at most $c$.

Proof. The conditions are clearly necessary. An application of Theorem 2 establishes their sufficiency.

Let $(E, F)$ be a dual pair and let $A$ be a non-empty $\sigma(E, F)$-bounded set. The $\sigma\left(F^{*}, F\right)$-closed absolutely convex envelope $B$ of $A$ is $\sigma\left(F^{*}, F\right)$ compact. Let $H$ be the linear span of $A$ and let $L$ be the linear span of $B$. $\left(F / H^{o}, L\right)$ is a dual pair and $F / H^{o}$ is a normed space under $\tau\left(F / H^{o}, L\right)$ with $B$ as the closed unit ball of the dual space $L$. We denote this normed space by $\mathcal{N}(F, A)$ and its completion by $\mathscr{B}(F, A)$. We now characterise essential separability for $A$ in terms of these spaces.

Lemma 1. If a normed space $E$ has a total subset $D$ with $|D| \leqslant c$, then $\operatorname{dim} E \leqslant c$.

Proof. The linear span $X$ of $D$ has cardinality at most $c$ and since each element of $E$ is the limit of a sequence in $X$, it follows that $|E| \leqslant c^{\aleph_{0}}=c$. Thus $\operatorname{dim} E \leqslant c$.

Theorem 3. Let $(E, F)$ be a dual pair and let $A$ be a non-empty $\sigma(E, F)$-bounded set. The following are equivalent:

(i) $A$ is essentially separable;

(ii) $\operatorname{dim} \mathcal{N}(F, A) \leqslant c$;

(iii) $\operatorname{dim} \mathscr{B}(F, A) \leqslant c$.

Proof. The argument used in the first part of the proof of Theorem 1 shows that (i) $\Rightarrow$ (ii), for $\mathcal{N}(F, A)^{*}$ is isomorphic to the $\sigma\left(F^{*}, F\right)$-closed linear span of $A$. ((ii) $\Rightarrow$ (iii)) follows from Lemma 1 , while ((iii) $\Rightarrow$ (ii)) is trivial.

Suppose that (ii) holds. The $\sigma\left(F^{*}, F\right)$-closed linear span of $A$ is $\sigma\left(F^{*}, F\right)$-separable, being isomorphic to a product of at most $c$ copies of the scalar field (4, Chapter VIII, Theorem 7.2). Thus $A$ is essentially separable.

As a corollary we have a partial converse of Theorem 1.

Corollary. Let $A$ be a non-empty absolutely convex $\sigma(E, F)$-bounded 
set. Then $A$ is essentially separable if and only if 0 has a base of neighbourhoods for $\left.\sigma(E, F)\right|_{A}$ consisting of at most $c$ sets.

Proof. The necessity of the condition is immediate by Theorem 1 .

If the condition is satisfied, there is a set $\left\{\phi_{\lambda}: \lambda \in \Lambda\right\}$ of non-empty finite subsets of $F$ such that $|\Lambda| \leqslant c$ and $\left\{\left\{x \in A:\left|\left\langle x, x^{\prime}\right\rangle\right| \leqslant 1, x^{\prime} \in \phi_{\lambda}\right\}: \lambda \in \Lambda\right\}$ is a base of neighbourhoods of 0 for $\left.\sigma(E, F)\right|_{A}$. The $\sigma\left(F^{*}, F\right)$-closure $B$ of $A$ is $\sigma\left(F^{*}, F\right)$-compact and absolutely convex, and by the bipolar theorem $\left\{\left\{x \in B:\left|\left\langle x, x^{\prime}\right\rangle\right| \leqslant 1, x^{\prime} \in \phi_{\lambda}\right\}: \lambda \in \Lambda\right\}$ is a base of neighbourhoods of 0 under $\left.\sigma\left(F^{*}, F\right)\right|_{B}$.

Let $z \in B \backslash\{0\}$. Then there exists $\lambda_{0} \in \Lambda$ such that $\left|\left\langle z, x^{\prime}\right\rangle\right|>1$ for some $x^{\prime} \in \phi_{\lambda_{0}}$. It follows that $D=\cup\left\{\phi_{\lambda}: \lambda \in \Lambda\right\}$ separates the elements of $B$ and so the set of equivalence classes in $\mathcal{N}(F, A)$ of the elements of $D$ is total. Since $|D| \leqslant c$, the result now follows from Lemma 1 and Theorem 3 .

Remark. The Corollary to Theorem 1 does not have a similar converse. If $E=l_{\infty}^{\prime \prime}$ and $F=l_{\infty}^{\prime}$, the closed unit ball $A$ of $l_{\infty}$ is a $\sigma\left(l_{\infty}^{\prime \prime}, l_{\infty}^{\prime}\right)$-dense subset of the closed unit ball $B$ of $l_{\infty}^{\prime \prime}$ and $|A|=c$. Clearly $\mathcal{N}\left(l_{\infty}^{\prime}, A\right)=$ $\mathscr{B}\left(l_{\infty}^{\prime}, A\right) \simeq l_{\infty}^{\prime}$. Now it follows from (17, Theorem 2.3 and Note 1.8(a)) that $\operatorname{dim} l_{\infty}^{\prime} \geqslant 2^{c}$. In fact $\operatorname{dim} l_{\infty}^{\prime}=2^{c}$ for $\operatorname{dim} l_{\infty}=c$ and $\operatorname{dim} l_{\infty}^{\prime} \leqslant \operatorname{dim} l_{\infty}^{*}=c^{c}=2^{c}$. Thus neither $A$ nor $B$ is essentially separable for the dual pair $\left(l_{\infty}^{\prime \prime}, l_{\infty}^{\prime}\right)$. Note however that $A$ is essentially separable for the dual pair $\left(l_{\infty}, l_{1}\right)$.

We now identify some particular essentially separable sets.

Lemma 2. Let $E$ be a normed space and let $B$ be the closed unit ball of $E^{\prime}$. If $|B|=c$ then $\operatorname{dim} E \leqslant c$.

Proof. There is a set $\Lambda$ with cardinality at most $c$ and a bijection $\lambda \mapsto x_{\lambda}^{\prime}$ of $\Lambda$ onto $A=\left\{x^{\prime} \in B:\left\|x^{\prime}\right\|=1\right\}$. For each $\lambda \in \Lambda$, choose $x_{\lambda} \in E$ such that $\left\langle x_{\lambda}, x_{\lambda}^{\prime}\right\rangle \neq 0$. Let $M$ be the closed vector subspace of $E$ generated by $\left\{x_{\lambda}: \lambda \in \Lambda\right\}$. Then $M=E$, for otherwise we would be able to find $\lambda_{0} \in \Lambda$ such that $\left\langle x, x_{\lambda_{0}}^{\prime}\right\rangle=0$ for all $x \in M$, contradicting $\left\langle x_{\lambda_{0}}, x_{\lambda_{0}}^{\prime}\right\rangle \neq 0$.

The result now follows from Lemma 1 .

Corollary. Let $(E, F)$ be a dual pair and let $A$ be a $\sigma(E, F)$-compact convex set. If $|A| \leqslant c$ then $A$ is essentially separable.

Proof. Let $C$ be the balanced hull of $A$ and let $B$ be the closed absolutely convex envelope of $A$. Then $C$ is $\sigma(E, F)$-compact, $|C| \leqslant c$ and since $B \subseteq C+C+C+C$, it follows that $B$ is a $\sigma(E, F)$-compact set with cardinality at most $c$. In fact $|B|=0,1$ or $c$. In the case $|B|=c$ the corollary now follows from Lemma 2 and Theorem 3. The other cases are trivial.

Theorem 4. Let $E$ be a separated locally convex space whose topology is defined by at most $c$ seminorms and let $F$ be the completion of $E$. Then each 
subset of $E$ which is $\sigma\left(F, E^{\prime}\right)$-relatively compact and whose cardinality is at most $c$ is essentially separable.

Proof. Since the topology of $F$ is also defined by at most $c$ seminorms and since the dual of $F$ is (isomorphic to) $E^{\prime}$, it is enough to establish the result when $E$ is complete. We may regard $E$ as a subspace of a product $\Pi\left\{E_{\lambda}: \lambda \in \Lambda\right\}$ of Banach spaces $E_{\lambda}(\lambda \in \Lambda)$ where $|\Lambda| \leqslant c$ (13, Chapter V, Proposition 16 and Corollary to Proposition 19). For each $\lambda \in \Lambda$ let $p_{\lambda}$ be the canonical projection of the product onto $E_{\lambda}$.

Let $A$ be a non-empty $\sigma\left(E, E^{\prime}\right)$-relatively compact set with $|A| \leqslant c$. For each $\lambda \in \Lambda, p_{\lambda}(A)$ is $\sigma\left(E_{\lambda}, E_{\lambda}^{\prime}\right)$-relatively compact and so by Krein's theorem (10, Section 24, 5(4)) the $\sigma\left(E_{\lambda}, E_{\lambda}^{\prime}\right)$-closed absolutely convex envelope $B_{\lambda}$ of $p_{\lambda}(A)$ is $\sigma\left(E_{\lambda}, E_{\lambda}^{\prime}\right)$-compact. Now $\left|p_{\lambda}(A)\right| \leqslant c$ and so the absolutely convex envelope $D_{\lambda}$ of $p_{\lambda}(A)$ has cardinality at most $c$. Since each element of $B_{\lambda}$ is the $\sigma\left(E_{\lambda}, E_{\lambda}^{\prime}\right)$-limit of a sequence in $D_{\lambda}(10$, Section $24,1(7))$, it is easily shown that $\left|B_{\lambda}\right| \leqslant c$. By the Corollary to Lemma $2, B_{\lambda}$ and therefore $p_{\lambda}(A)$ are essentially separable for the dual pair $\left(E_{\lambda}, E_{\lambda}^{\prime}\right)$. Let $C_{\lambda}$ be a $\sigma\left(E_{\lambda}^{\prime *}, E_{\lambda}^{\prime}\right)$-separable set which contains $p_{\lambda}(A)$. We now have

$$
A \subseteq \prod\left\{p_{\lambda}(A): \lambda \in \Lambda\right\} \subseteq C=\prod\left\{C_{\lambda}: \lambda \in \Lambda\right\}
$$

and $C$ is $\sigma\left(\Pi_{\lambda \in \Lambda} E_{\lambda}^{\prime *}, \Sigma_{\lambda \in \Lambda} E_{\lambda}^{\prime}\right)$-separable (4, Chapter VIII, Theorem 7.2). Thus $A$ is essentially separable for the dual pair $\left(\prod_{\lambda \in \Lambda} E_{\lambda}, \Sigma_{\lambda \in \Lambda} E_{\lambda}^{\prime}\right)$.

Let $H$ be the linear span of $A$ and let $H^{\bullet}, E^{\bullet}$ be the polars of $H$ and $E$ respectively in $\Sigma_{\lambda \in \Lambda} E_{\lambda}^{\prime}$ and let $H^{\bullet}$ be the polar of $H$ in $E^{\prime}$. Then

$$
\left(\sum_{\lambda \in \Lambda} E_{\lambda}^{\prime}\right) / H^{\bullet} \simeq\left(\left(\sum_{\lambda \in \Lambda} E_{\lambda}^{\prime}\right) / E^{\bullet}\right) /\left(H^{\bullet} / E^{\bullet}\right) \simeq E^{\prime} / H^{\bullet} \text {. }
$$

The result now follows from Theorem 3 .

Corollary. Let $E, F$ be as in the theorem and let $B$ be a subset of $E$ which is $\sigma\left(F, E^{\prime}\right)$-relatively compact. If $x$ is an element of the $\sigma\left(F, E^{\prime}\right)-$ closure of $B$, there is an essentially separable subset $A$ of $B$ such that $x$ is in the $\sigma\left(F, E^{\prime}\right)$-closure of $A$.

Proof. By (16, (b)) there is a subset $A$ of $B$ with cardinality at most $c$ such that $x$ is in the $\sigma\left(F, E^{\prime}\right)$-closure of $A$. The result now follows from the theorem since $A$ is also $\sigma\left(F, E^{\prime}\right)$-relatively compact.

As an application of this corollary we obtain in Theorem 5 criteria for weak compactness and weak relative compactness in a separated locally convex space whose topology is defined by at most $c$ seminorms. These would appear to be the natural analogues of the well-known sequential criteria in a metrizable locally convex space (10, Section $24,3(8)$, (9)).

Theorem 5. Let $E$ be a separated locally convex space whose topology 20/4-D 
is defined by at most $c$ seminorms. $A$ subset $B$ of $E$ is $\sigma\left(E, E^{\prime}\right)$-relatively compact (resp. $\sigma\left(E, E^{\prime}\right)$-compact) if and only if each essentially separable subset of $B$ is $\sigma\left(E, E^{\prime}\right)$-relatively compact (resp. $\sigma\left(E, E^{\prime}\right)$-relatively compact and has its $\sigma\left(E, E^{\prime}\right)$-closure contained in $\left.B\right)$.

Proof. The conditions are clearly necessary.

Under either condition, $B$ is $\sigma\left(E, E^{\prime}\right)$-relatively countably compact, since a countable set is trivially essentially separable. Then if $F$ is the completion of $E, B$ is $\sigma\left(F, E^{\prime}\right)$-relatively compact by Eberlein's theorem (10, Section 24, 2(1)). It follows from the Corollary to Theorem 4 that each $\sigma\left(F, E^{\prime}\right)$-point of closure $x$ of $B$ is already in $E$ so that $B$ is $\sigma\left(E, E^{\prime}\right)$ relatively compact under either condition. Under the bracketed condition, $x \in B$ so that $B$ is $\sigma\left(E, E^{\prime}\right)$-compact.

The concept of Schauder dimension for Banach spaces was introduced in (7). We end the present section by combining this idea with a property of essentially separable sets. The terminology is that of (7).

Theorem 6. Let $E$ be a Banach space and suppose that every subset of the closed unit ball of $E^{\prime}$ has a $\sigma\left(E^{\prime}, E\right)$-dense subset of cardinality at most c. If $E$ has a Schauder dimension, then $\operatorname{dim} E \leqslant c$.

Proof. Let $\left\{x_{\lambda}: \lambda \in \Lambda\right\}$ be a maximal strongly linearly independent subset of $E$ and let $\left\{x_{\lambda}^{\prime}: \lambda \in \Lambda\right\}$ be a subset of $E^{\prime}$ such that $\left\langle x_{\mu}, x_{\lambda}^{\prime}\right\rangle=\delta_{\lambda \mu}$ for all $\lambda, \mu \in \Lambda$. Now $\left\{\left\|x_{\lambda}^{\prime}\right\|^{-1} x_{\lambda}^{\prime}: \lambda \in \Lambda\right\}$ is a subset of the closed unit ball of $E^{\prime}$ with no proper $\sigma\left(E^{\prime}, E\right)$-dense subset, for if $\lambda \neq \mu,\left\langle x_{\lambda},\left\|x_{\lambda}^{\prime}\right\|^{-1} x_{\lambda}^{\prime}-\left\|x_{\mu}^{\prime}\right\|^{-1} x_{\mu}^{\prime}\right\rangle=$ $\left\|x_{\lambda}^{\prime}\right\|^{-1}$. Thus $|\Lambda| \leqslant c$. Since $\left\{x_{\lambda}: \lambda \in \Lambda\right\}$ is total in $E$ (7, Proposition 1), the result now follows from Lemma 1 .

\section{3. $\delta$-barrelled spaces}

We gave the following definition in (12).

A separated locally convex space $E$ is $\delta$-barrelled if each essentially separable $\sigma\left(E^{\prime}, E\right)$-bounded set is equicontinuous.

We showed by example that a $\delta$-barrelled space need not be barrelled even in its associated Mackey topology. On the other hand, since $\delta$ barrelled spaces are necessarily $\sigma$-barrelled, a separable $\delta$-barrelled space is barrelled (3, Corollary $4 a)$. Further a $\delta$-barrelled space which has a strongly dense subset of cardinality at most $c$ is always barrelled (12, Corollary 2 of Theorem 3 ). We now give two generalisations of this last result.

Theorem 7. Let $E$ be a $\delta$-barrelled space with completion $F$. Suppose that there is a family $\left(X_{\lambda}\right)_{\lambda \in \Lambda}$ of subsets of $E$ such that

(i) $|\Lambda| \leqslant c$, 
(ii) $\cup\left\{X_{\lambda}: \lambda \in \Lambda\right\}$ is total in $E$ under $\beta\left(E, E^{\prime}\right)$,

(iii) for each $\lambda \in \Lambda, X_{\lambda}$ is $\sigma\left(F, E^{\prime}\right)$-relatively compact.

Then $\left(E, \tau\left(E, E^{\prime}\right)\right)$ is barrelled.

Proof. Let $Y_{\lambda}$ be the $\sigma\left(F, E^{\prime}\right)$-closed absolutely convex envelope of $X_{\lambda}$ $(\lambda \in \Lambda)$. By Krein's theorem (10, Section 24, 5(4)) and (iii), each $Y_{\lambda}$ is $\sigma\left(F, E^{\prime}\right)$-compact. Denote by $G$ the subspace of $F$ spanned by $\cup\left\{Y_{\lambda}: \lambda \in\right.$ $\Lambda\}$ and by $H$ the subspace of $E$ spanned by $\cup\left\{X_{\lambda}: \lambda \in \Lambda\right\}$.

Let $B$ be a $\sigma\left(E^{\prime}, E\right)$-closed bounded set and let $A$ be a subset of $B$ which is essentially separable for the dual pair $\left(E^{\prime}, G\right)$. Certainly $A$ is essentially separable for the dual pair $\left(E^{\prime}, H\right)$ and since $H$ is $\beta\left(E, E^{\prime}\right)$ dense in $E$, it follows easily from Theorem 3 that $A$ is essentially separable for the dual pair $\left(E^{\prime}, E\right)$. Since $E$ is $\delta$-barrelled, $A$ is equicontinuous. If $C$ is the $\sigma\left(E^{\prime}, E\right)$-closure of $A$, then $C \subseteq B$ and $C$ is also $\sigma\left(E^{\prime}, F\right)$-compact (13, Chapter VI, Corollary 3 of Theorem 2) and therefore $\sigma\left(E^{\prime}, G\right)$ compact. Since each $Y_{\lambda}$ is $\sigma\left(G, E^{\prime}\right)$-compact and absolutely convex, $E^{\prime}$ has a topology of the dual pair $\left(E^{\prime}, G\right)$ defined by at most $c$ seminorms and so by Theorem $5, B$ is $\sigma\left(E^{\prime}, G\right)$-compact. This implies that $B$ is $\sigma\left(E^{\prime}, H\right)$ compact, and since $E$ is contained in the completion of $H$ for the topology induced by $\beta\left(E, E^{\prime}\right)$, we deduce that $B$ is $\sigma\left(E^{\prime}, E\right)$-compact.

Remark. In Theorem 7, the initial $\delta$-barrelled topology of $E$ need not be $\tau\left(E, E^{\prime}\right)$. To see this, we refer to $(12$, Theorem 2 and Remark (i) following Theorem 8). If $|M|>c, l_{2}(M)$ is $\delta$-barrelled but not barrelled under the topology of uniform convergence on the $\sigma\left(l_{2}(M), l_{2}(M)\right)$-bounded essentially separable sets. However the conditions of Theorem 7 are satisfied by taking the closed unit ball of $l_{2}(M)$ as the single $X_{\lambda}$.

We require the following lemma for our other result in this direction. It is probably well-known but we include a proof for completeness.

Lemma 3. Let $E$ be a $\sigma$-barrelled space. If $\Sigma_{\lambda \in \Lambda} x_{\lambda}$ converges unconditionally in $E$, it also converges unconditionally under $\beta\left(E, E^{\prime}\right)$ to the same sum.

Proof. It is enough to show that $\Sigma_{\lambda \in \Lambda} x_{\lambda}$ is unconditionally Cauchy under $\beta\left(E, E^{\prime}\right)$, for then the result will follow from $(10$, Section 18, 4(4)). Suppose that this is false and denote by $\Phi$ the set of all non-empty finite subsets of $\Lambda$. Then there is a $\sigma\left(E^{\prime}, E\right)$-bounded set $B$ such that for each $\phi \in \Phi$, there exist $\phi^{\prime} \in \Phi$ with $\phi^{\prime} \cap \phi=\emptyset$ and $x^{\prime} \in B$ such that $\left|\left\langle\Sigma_{\lambda \in \phi^{\prime}} x_{\lambda}, x^{\prime}\right\rangle\right|>1$. We can thus determine sequences $\left(\phi_{n}\right)$ in $\Phi$ and $\left(x_{n}^{\prime}\right)$ in $B$ such that $\phi_{n+1} \cap \cup_{r=1}^{n} \phi_{r}=\emptyset$ and $\left|\left\langle\sum_{\lambda \in \phi_{n}} x_{\lambda}, x_{n}^{\prime}\right\rangle\right|>1(n \in N)$.

But $\left\{x_{n}^{\prime}: n \in N\right\}$ is equicontinuous and so there exists $\phi_{0} \in \Phi$ such that $\left|\left\langle\Sigma_{\lambda \in \phi} x_{\lambda}, x_{n}^{\prime}\right\rangle\right| \leqslant 1$ for all $n \in N$ and for all $\phi \in \Phi$ with $\phi \cap \phi_{0}=\emptyset$. Since $\phi_{n} \cap \phi_{0}=\emptyset$ for all sufficiently large $n$ we obtain a contradiction. 
Theorem 8. Let $E$ be a $\delta$-barrelled space and suppose that there is a family $\left(x_{\mu}\right)_{\mu \in M}$ of elements of $E$ such that

(a) for each $x \in E$ there exist scalars $\alpha_{\mu}(\mu \in M)$ such that $\Sigma \alpha_{\mu} x_{\mu}$ is unconditionally convergent to $x$,

(b) there is a family $\left(z_{\lambda}\right)_{\lambda \in \Lambda}$ of elements of $E$ such that $z_{\lambda}=\Sigma \alpha_{\mu}^{(\lambda)} x_{\mu}$ $(\lambda \in \Lambda),|\Lambda| \leqslant c$ and for each $\mu \in M$, at least one $\alpha_{\mu}^{(\lambda)} \neq 0$.

Then each $\sigma\left(E^{\prime}, E\right)$-bounded set is essentially separable and consequently $E$ is barrelled.

Proof. Let $A$ be a non-empty $\sigma\left(E^{\prime}, E\right)$-bounded set. For each $x \in E$ let $\bar{x}$ denote its equivalence class in $\mathcal{N}(E, A)$. If $x=\sum \alpha_{\mu} x_{\mu}$ as above, it follows from Lemma 3 that $\sum \alpha_{\mu} \bar{x}_{\mu}$ converges unconditionally to $\bar{x}$ in $\mathcal{N}(E, A)$. Since $\mathcal{N}(E, A)$ is a normed space, $\left\{\mu \in M: \alpha_{\mu} \bar{x}_{\mu} \neq 0\right\}$ is at most countable and so $\cup_{\lambda \in \Lambda}\left\{\mu \in M: \alpha_{\mu}^{(\lambda)} \bar{x}_{\mu} \neq 0\right\}$ has cardinality at most $c$. But by (b) this set is just $\left\{\mu \in M: \bar{x}_{\mu} \neq 0\right\}$. Since $\left\{\bar{x}_{\mu}: \mu \in M\right\}$ is total in $\mathcal{N}(E, A)$ the result now follows from Lemma 1 and Theorem 3.

Remark. It should be noted that the space $E$ of Theorem 8 need not have a dense subset of cardinality at most $c$. Using the argument in part (3) of the proof of (4, Chapter VIII, Theorem 7.2), we see that $\mathbb{R}^{M}$ has no such subset if $|M|>2^{c}$. However we may apply Theorem 8 to $\mathbb{R}^{M}$ with $x_{\mu}=$ $\left(\delta_{\mu \gamma}\right)_{\gamma \in M}$ and a single $z_{\lambda}$, viz $\Sigma x_{\mu}$. Theorem 8 is an analogue of (15, Theorem 1).

In (12, Theorem 2) we showed that a $\delta$-barrelled space $E$ is both $\delta$-barrelled and countably barrelled (8) under the topology $\delta\left(E, E^{\prime}\right)$ of uniform convergence on the $\sigma\left(E^{\prime}, E\right)$-bounded essentially separable sets. We end this section by giving an example of a $\delta$-barrelled space which is not countably barrelled. In (14, Proposition 4.4), J. Schmets describes a general method of constructing $\sigma$-barrelled spaces which are not countably barrelled. We adapt this technique to our present purpose, although our approach is rather different.

Let $E=\mathbb{R}^{(M)}$ and let $E^{\prime}=\left\{\left(\xi_{\mu}\right) \in \mathbb{R}^{M}:\left|\left\{\mu: \xi_{\mu} \neq 0\right\}\right| \leqslant c\right\}$. For any subset $A$ of $E^{\prime}$ let $\operatorname{supp} A=\left\{\nu \in M: \exists\left(\xi_{\mu}\right) \in A\right.$ with $\left.\xi_{\nu} \neq 0\right\}$. It follows from the Corollary to Theorem 1 that if $A$ is essentially separable for the dual pair $\left(E^{\prime}, E\right),|\operatorname{supp} A| \leqslant c(*)$. Thus if $A$ is a $\sigma\left(E^{\prime}, E\right)$-bounded essentially separable set, it is $\sigma\left(E^{\prime}, E\right)$-relatively compact. Since the closed absolutely convex envelope of an essentially separable set is essentially separable, the Mackey-Arens theorem shows that $\delta\left(E, E^{\prime}\right)$ is a topology of the dual pair ( $E, E^{\prime}$ ) under which $E$ is $\delta$-barrelled (cf. Example 1 of (12)).

For each non-empty subset $B$ of $M$

$$
S(B)=\left\{\left(\xi_{\mu}\right) \in \mathbb{R}^{M}: \xi_{\mu}=0 \text { if } \mu \notin B, \Sigma\left|\xi_{\mu}\right| \leqslant 1\right\}
$$

is easily seen to be a closed bounded absolutely convex subset of $\mathbb{R}^{M}$. 
Therefore $S(B)$ is compact in $\mathbb{R}^{M}$ and since it is contained in $E^{\prime}$, it is $\sigma\left(E^{\prime}, E\right)$-compact.

We now take $M=\mathscr{P}(\mathrm{R})$, the power set of $\mathrm{R}$. In this case $E^{\prime} \neq \mathrm{R}^{M}$ and $\left(E, \tau\left(E, E^{\prime}\right)\right)$ is not barrelled. Let $\mathscr{B}$ be the collection of all $\sigma\left(E^{\prime}, E\right)$ bounded essentially separable sets together with the sets $S(\mathscr{P}(C)$ ) where $C$ is a compact subset of $\mathrm{R}$. The topology $\xi$ on $E$ of uniform convergence on the sets in $\mathscr{B}$ is then a $\delta$-barrelled topology of the dual pair $\left(E, E^{\prime}\right)$ and a base of neighbourhoods of the origin for $\xi$ is given by all sets of the form $D^{\circ} \cap \epsilon S(\mathscr{P}(C))^{\circ}(* *)$, where $D$ is a non-empty $\sigma\left(E^{\prime}, E\right)$-bounded essentially separable set, $\epsilon>0$ and $C$ is a compact subset of $R$.

Now $A=\cup_{n=1}^{\infty} S(\mathscr{P}([-n, n]))$ is a subset of $S(\mathscr{P}(\mathbb{R}))$ so that $A$ is a $\sigma\left(E^{\prime}, E\right)$-bounded set which is the union of a sequence of $\xi$-equicontinuous sets. Given any set $V$ of the form $(* *)$, by $(*)$ and the fact that $|\mathscr{P}([-n, n])| \mathscr{P}(C) \mid=2^{c}$ for all sufficiently large $n$, we may choose $\nu \in$ $\left(\cup_{n=1}^{\infty} \mathscr{P}([-n, n])\right) \backslash\{(\operatorname{supp} D) \cup \mathscr{P}(C)\}$. Then $\left(2 \delta_{\mu \nu}\right)_{\mu \in M} \in V$ so that $\left(\delta_{\mu \nu}\right)_{\mu \in M}$ $\notin V^{\circ}$. Since $\left(\delta_{\mu \nu}\right)_{\mu \in M} \in A$, this shows that $A$ is not $\xi$-equicontinuous and consequently $(E, \xi)$ is $\delta$-barrelled but not countably barrelled.

\section{Infra- $\delta$-spaces}

Let $E$ be a separated locally convex space and for each vector subspace $H$ of $E^{\prime}$ let $H^{\delta}$ be the intersection of all vector subspaces $G$ of $E^{*}$ such that

(i) $H \subseteq G$,

(ii) the $\sigma\left(E^{*}, E\right)$-closure of each $\sigma\left(E^{*}, E\right)$-bounded subset of $G$ which is essentially separable for the dual pair $\left(E^{*}, E\right)$ is contained in $G$.

As in (12) we say that $E$ is an infra- $\delta$-space if for each $\sigma\left(E^{\prime}, E\right)$-dense vector subspace $H$, we have $E^{\prime} \cap H^{\delta}=E^{\prime}$.

For any separated locally convex space $E$, the upper bound topology $\eta$ of the initial topology $\xi$ of $E$ and $\delta\left(E,\left(E^{\prime}\right)^{\delta}\right)$ is clearly the coarsest $\delta$-barrelled topology on $E$ which is finer than $\xi$. We call $\eta$ the associated $\delta$-barrelled topology of $E$. This definition is analogous to Adasch's definition of the associated barrelled topology (1), which is clearly finer than the associated $\delta$-barrelled topology.

It is shown in (5, Theorem 1.5) and in (2, Section 4) that an infra- $s$ space (1) is complete in its associated barrelled topology. As pointed out in (12), the infra- $\delta$-spaces form a proper subclass of the infra- $s$-spaces, so that this completeness result applies to infra- $\delta$-spaces. However essentially the same proof as that given in (5) shows that an infra- $\delta$-space is actually complete in its associated $\delta$-barrelled topology. To show that this is a genuine improvement, we adapt ideas from (6) to give an example of an infra- $\delta$-space for which the associated barrelled topology and the as- 
sociated $\delta$-barrelled topology are not even topologies of the same dual pair.

Let $E=\mathbf{R}^{M}$ where $|M|=2^{c}$ and let $E^{\prime}=\left\{\left(\xi_{\mu}\right) \in \mathbf{R}^{M}:\left|\left\{\mu: \xi_{\mu} \neq 0\right\}\right| \leqslant \aleph_{0}\right\}$. We show first of all that $E$ is an infra- $\delta$-space for any topology of the dual pair $\left(E, E^{\prime}\right)$. Let $H$ be a $\sigma\left(E^{\prime}, E\right)$-dense vector subspace and let $\left(x_{n}^{\prime}\right)$ be a sequence in $H^{\delta} \cap E^{\prime}$ which converges to $x^{\prime} \in E^{\prime}$ under $\sigma\left(E^{\prime}, E\right)$. Since $\left\{x_{n}^{\prime}: n \in N\right\}$ is (essentially) separable, its $\sigma\left(E^{*}, E\right)$-closure must be contained in each $G$ considered in constructing $H^{\delta}$. Thus $x^{\prime} \in H^{\delta}$ and so $H^{\delta} \cap E^{\prime}$ is $\sigma\left(E^{\prime}, E\right)$-sequentially closed. But as pointed out by V. Eberhardt in (6), Theorem 2.1 of (11) now shows that $H^{\delta} \cap E^{\prime}$ is $\sigma\left(E^{\prime}, E\right)$ closed. Since $H \subseteq H^{\delta} \cap E^{\prime}$, we must then have $H^{\delta} \cap E^{\prime}=E^{\prime}$.

It is clear that if $B$ is any subset of $\mathbb{R}^{M}$ which is a product of intervals, $B \cap E^{\prime}$ is $\sigma\left(\mathbf{R}^{M}, \mathbf{R}^{(M)}\right)$-dense in $B$. It follows from this observation that the associated barrelled topology for any topology of the dual pair $\left(E, E^{\prime}\right)$ is $\tau\left(\mathbf{R}^{(M)}, \mathbf{R}^{M}\right)$. However if $F^{\prime}=\left\{\left(\xi_{\mu}\right) \in \mathbf{R}^{M}:\left|\left\{\mu: \xi_{\mu} \neq 0\right\}\right| \leqslant c\right\}$, we know from the previous section that $\delta\left(E, F^{\prime}\right)$ is a $\delta$-barrelled topology of the dual pair $\left(E, F^{\prime}\right)$. If we start with the topology $\sigma\left(E, E^{\prime}\right)$ on $E$, the associated $\delta$-barrelled topology $\eta$ must therefore be coarser than $\delta\left(E, F^{\prime}\right)$. (In fact it is not difficult to show that $\eta=\delta\left(E, F^{\prime}\right)$ ). Since $F^{\prime} \neq \mathbb{R}^{M}$, we may take $\left(E, \sigma\left(E, E^{\prime}\right)\right)$ for the promised example.

\section{REFERENCES}

(1) N. Adasch, Tonnelierte Räume und zwei Sätze von Banach, Math. Ann. 186 (1970), 209-214.

(2) N. ADASCH, Vollständigkeit und der Graphensatz, J. Reine Angew. Math. 249 (1971), 217-220.

(3) M. DE WILDE and C. HoUET, On increasing sequences of absolutely convex sets in locally convex spaces, Math. Ann. 192 (1971), 257-261.

(4) J. Dugundu, Topology (Allyn and Bacon, Boston, 1966).

(5) V. EBERHARDT, Der Graphensatz von A.P. und W. Robertson für $s$ Räume, Manuscripta Math. 4 (1971), 255-262.

(6) V. EBERHARDT, Einige Vererbbarkeitseigenschaften von $B$-und $B_{r}$-vollständigen Räumen, Math. Ann. 215 (1975), 1-11.

(7) J. W. Evans and R. A. TAPIA, Hamel versus Schauder dimension, Amer. Math. Monthly 77 (1970), 385-388.

(8) T. Husain, Two new classes of locally convex spaces, Math. Ann. 166 (1966), 289-299.

(9) N. J. Kalton, Some forms of the closed graph theorem, Proc. Cambridge Philos. Soc. 70 (1971), 401-408.

(10) G. KöTHE, Topological Vector Spaces I (Springer-Verlag, Berlin, 1969). 
(11) N. NOBLE, The continuity of functions on cartesian products, Trans. Amer. Math. Soc. 149 (1970), 187-198.

(12) J. O. POPOOLA and I. TWEdDLE, On the closed graph theorem, Glasgow Math. J. 17(1976), 89-97.

(13) A. P. RobertSON and W. J. ROBERTSON, Topological Vector Spaces, 2nd edition (Cambridge University Press, Cambridge, 1973).

(14) J. SCHMETS, Espaces associés à un espace linéaire à semi-normes-applications aux espaces de fonctions continues (Université de Liège, 1972-3).

(15) I. TwedDle, Unconditional convergence and bases, Proc. Edinburgh Math. Soc. 18 (Series II) (1973), 321-324.

(16) M. VAldivia, Some criteria for weak compactness, J. Reine Angew. Math. 255 (1972), 165-169.

(17) K. Yosida and E. HEwITT, Finitely additive measures, Trans. Amer. Math. Soc. 72 (1952), 46-66.

UNIVERSITY OF STIRLING

AND

UNIVERSITY OF LAGOS 